\title{
RESEÑA. TECNOLOGÍAS DE LA INFORMACIÓN Y LA COMUNICACIÓN EN CONTEXTOS EDUCATIVOS
}

\section{REVIEW. TECNOLOGÍAS DE LA INFORMACIÓN Y LA COMUNICACIÓN EN CONTEXTOS EDUCATIVOS}

\section{RESENHA. TECNOLOGÍAS DE LA INFORMACIÓN Y LA COMUNICACIÓN EN CONTEXTOS EDUCATIVOS}

\author{
Alejandro Colomo Magaña \\ Universidad de Málaga, España \\ https://orcid.org/0000-0002-8472-5652 \\ alejandro.c.m.91@hotmail.com
}

Recibido: 24/09/2021 Revisado:15/10/2021 Aceptado: 15/10/2021 Publicado: 26/10/2021

Autor: Enrique Sánchez Rivas, Ernesto Colomo Magaña, y Julio Ruiz Palmero (Coords).

Editorial: Síntesis

Año: 2020

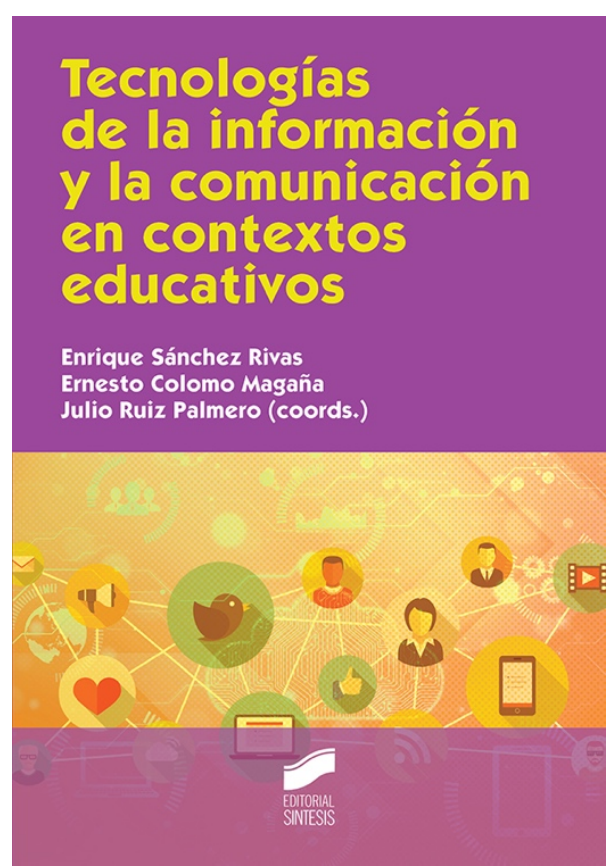

Cómo citar esta reseña: Colomo, A. (2021). Reseña. Tecnologías de la información y la comunicación en contextos educativos. Hachetetepé. Revista cientifica en Educación y Comunicación, (23), 1-3. https://doi.org/10.25267/Hachetetepe.2021.i23.2302

En plena era digital, donde los seres humanos viven conectados y las fronteras son cada vez más difusas en una realidad hiperconectada, las tecnologías suponen el pilar clave para una nueva forma de comunicarnos, relacionarnos, aprender y de vivir en sociedad. Un contexto cada vez más accesible e interactivo que multiplica nuestras posibilidades como personas, incluidos ámbitos como la cultura, la economía o la educación. Es en este campo donde se enmarca la obra coordinada por los docentes 
Enrique Sánchez Rivas, Ernesto Colomo Magaña y Julio Ruiz Palmero, pertenecientes a la Universidad de Málaga. El propósito general es dar a conocer el potencial que las tecnologías pueden reportar a los diferentes escenarios educativos (presenciales, híbridos, a distancia o e-Learning), tanto como soporte de los procesos como herramientas para el diseño y desarrollo de los procesos formativos. Aunando la visión y conocimientos de un grupo variados de expertos en tecnología educativa, tanto nacionales como internacionales, se abordan diferentes fundamentos de la tecnología educativa en los 14 capítulos que conforman la obra.

El libro se estructura en 3 apartados en función de las temáticas que se abordan en cada uno de los mismos.

En el primer apartado se ahonda en el impacto de las TIC y su papel dentro del terreno educativo, englobando desde el capítulo 1 hasta el 6. Si queremos que las tecnologías formen parte de los procesos de enseñanza, es fundamental que exista un desarrollo en las competencias digitales de los docentes encargados de la tarea educativa. No podemos aspirar a formar con tecnologías si los que deben explicar tienen carencias formativas, dudas o dificultades no solo para su uso, sino para poder resolver las cuestiones que se plantee su alumnado. Junto a ello, se resaltan, entre sus infinitas posibilidades académicas, la creación de contenido digital (plataformas, cursos de carácter online o materiales para las diferentes asignaturas), haciendo también uso de los mass media (por el interés que despiertan estos recursos entre las personas por sus diferentes configuraciones y propósitos y por su vinculación con la realidad sociocultural), para todo tipo de estudiantes, incluidos aquellos que presentan dificultades y/o necesidades para el aprendizaje.

El segundo apartado, que incluye los capítulos del 7 al 12, se centra en los recursos y herramientas tecnológicas y cómo estas impactan en el proceso educativo al ser utilizadas en clase. Cabe destacar el rol que adquieren los vídeos, como medio para la transmisión de contenidos de forma audiovisual, logrando captar la atención y aumentando la motivación del alumnado. También el convencimiento, cada vez mayor, de utilizar las redes sociales con fines didácticos. Estamos ante elementos que permiten consumir información por parte de los discentes, con enfoques diferentes de uso y posibilidades, convirtiéndose los vídeos cortos (denominados como píldoras formativas) en complementos a las clases magistrales (o sustitutorios en función de qué metodología se implemente), mientras que las redes sociales permiten el debate, la interacción y la reflexión sin límites temporales ni espaciales. Y es que el contexto digital, donde se apuesta por la autonomía del alumnado que conforma su propio entorno personal de aprendizaje, estos elementos formativos pueden ser seleccionados como recursos para abordar los temas de estudio, atendiendo a intereses y formas de aprender de cada discente. Unos recursos seleccionados que deben respetar la legalidad de uso de dichos materiales, profundizando en la importancia de las licencias Creative Commons. Para cerrar el tema de las herramientas digitales, sin que por ello sea menos relevante, se exponen posibilidades y beneficios de trabajar con la realidad aumentada mediante los teléfonos digitales, permitiendo conocer de forma más exhaustiva algunas propuestas tecnológicas con menor desarrollo en algunos contextos formativos.

El tercer y último apartado, que incluye los capítulos 13 y 14, ponen el foco en la metodología y la figura docente. Como refleja la obra, no se trata solo de incorporar tecnologías, sino de pensar los procesos, su estructura, organización, sentido y evaluación, incluyendo aquellas que respondan a dichos propósitos de la manera más 
eficiente y eficaz posible. Sumado a ello, la labor del docente adquiere un papel fundamental, pues es el responsable del diseño de las experiencias didácticas, de decidir cómo se va a trabajar en clase y de seleccionar aquellas herramientas más útiles, innovadoras y facilitadoras del aprendizaje de entre todas las que dispone a su acceso. El cometido es el desarrollo integral del educando, convirtiéndose la tecnología en un medio formativo para lograr dicha meta.

Podemos afirmar que la finalidad que persigue esta obra no solo es presentar las múltiples posibilidades que las TIC pueden ofrecer, dentro del contexto formativo, sino realizar un análisis pedagógico en el que se ponga de manifiesto su potencial como recurso educativo. No se trata de quedarse en la superficie y la correspondiente novedad e innovación, sino en profundizar en la esencia misma del hecho educativo, desgranando cómo la tecnología se convierte en medio (privilegiado) y no en un fin, en canal para enriquecer la labor de enseñanza y no como excusa sin fondo didáctico para incorporar la realidad digital al terreno escolar. En un contexto anterior al COVID-19, donde se enmarca la obra y las aportaciones de los autores, la reflexión sobre las tecnologías ya se fundamentaba en su visión complementaria y enriquecedora de los procesos. El modelo educativo desarrollado merced a las restricciones, con la consiguiente apuesta por modelos e-Learning e híbridos, ha evidenciado que el tránsito a lo tecnológico se ha construido sobre la inmediatez, siendo preciso una configuración metodológica de la formación mediada por tecnologías. Este libro, como un presentimiento sobre el devenir de la educación con TIC, apuesta por una revolución de los procesos, donde el educando se sitúa en el centro de un proceso educativo acompañado por tecnología para su desarrollo global y holístico.

\section{REFERENCIAS BIBLIOGRÁFICAS}

Sánchez-Rivas, E., Colomo-Magaña, E., y Ruiz-Palmero, J. (2020). Tecnologías de la información y la comunicación en contextos educativos. Síntesis. 